\title{
Escoliose degenerativa do adulto
}

\section{Adult Degenerative Scoliosis}

\author{
Alexandre Fogaça Cristante ${ }^{1}$ Ricardo Teixeira e Silva ${ }^{1}$ Guilherme Henrique Ricardo da Costa ${ }^{1}$ \\ Raphael Martus Marcon ${ }^{10}$ \\ 1 Departamento de Ortopedia e Traumatologia, Hospital das Clinicas, \\ Faculdade de Medicina, Universidade de São Paulo, São Paulo, SP, \\ Brasil \\ Rev Bras Ortop 2021;56(1):1-8.

\begin{abstract}
Endereço para correspondência Ricardo Teixeira e Silva, HCFMUSP, Faculdade de Medicina, Universidade de São Paulo, R. Dr. Ovídio Pires de Campos, 333, Cerqueira César, São Paulo, SP,
\end{abstract} \\ Departamento de Ortopedia e Traumatologia, Hospital das Clinicas \\ 05403-010, Brasil (e-mail: ricardo.teixeira.silva@gmail.com).
}

\section{Resumo \\ Palavras-chave \\ - escoliose/ fisiopatologia \\ - escoliose/terapia \\ - degeneração do disco intervertebral \\ - adulto}

O envelhecimento da população aumenta a incidência dos processos degenerativos osteomusculares, como a escoliose do adulto (EA). A EA é definida como uma deformidade da coluna no plano coronal com ângulo de Cobb $>10^{\circ}$ e pode ocorrer devido a um processo degenerativo (escoliose de novo), evolução de uma escoliose préexistente ou de forma iatrogênica.

A EA é uma doença potencialmente limitante que acomete um grupo heterogêneo de pacientes. O tratamento clínico se mostrou pouco efetivo e a indicação cirúrgica é frequente. No presente artigo, é apresentada uma revisão sobre a fisiopatologia, a manifestação clínica e o diagnóstico da EA. Também são apresentadas as indicações cirúrgicas e as principais técnicas utilizadas atualmente.

Populational aging increases the incidence of musculoskeletal degenerative processes, such as adult scoliosis (AS). Adult scoliosis is defined as a spinal deformity in the coronal plane with a Cobb angle $>10^{\circ}$. Adult scoliosis may be iatrogenic or result from a degenerative process (scoliosis de novo) or a pre-existing scoliosis.

Adult scoliosis is a potentially limiting condition that affects a heterogeneous group of patients. Clinical treatment proved to be ineffective and surgery is often indicated. The present paper reviews AS pathophysiology, clinical presentation and diagnosis, in addition to surgical indications and the main techniques currently used.

\section{Introdução}

A população brasileira encontra-se em processo de transição demográfica, com o envelhecimento de sua população. ${ }^{1}$ O grupo etário composto por pessoas $>65$ anos cresceu de 3,5\%, em 1970 para 5,5\% da população em 2000. Estima-se que corresponderá a $19 \%$ da população brasileira em $2050 .^{2}$

recebido

05 de Setembro de 2019

aceito

10 de Janeiro de 2020

Publicado online

Julho 22, 2020
Este fenômeno traz implicações importantes nos cuidados em saúde, sobretudo por aumentar a incidência e prevalência dos processos degenerativos osteomusculares, como a escoliose do adulto (EA). A prevalência de deformidades vertebrais em pessoas $>65$ anos varia de 32 a $68 \%$, em diferentes estudos. $^{3}$

A EA é definida como uma deformidade da coluna vertebral que apresenta um ângulo de $\mathrm{Cobb}>10^{\circ}$ no plano

(c) 2020. Sociedade Brasileira de Ortopedia e Traumatologia. All rights reserved.

This is an open access article published by Thieme under the terms of the Creative Commons Attribution-NonDerivative-NonCommercial-License, permitting copying and reproduction so long as the original work is given appropriate credit. Contents may not be used for commercial purposes, or adapted, remixed, transformed or built upon. (https://creativecommons.org/ licenses/by-nc-nd/4.0/)

Thieme Revinter Publicações Ltda., Rua do Matoso 170, Rio de Janeiro, RJ, CEP 20270-135, Brazil 
coronal, em um paciente esqueleticamente maduro. ${ }^{4}$ Ela pode ocorrer em razão do processo degenerativo da coluna (chamada de escoliose de novo), como progressão de uma escoliose pré-existente da infância/adolescência (escoliose idiopática do adulto [EIA]) ou de forma secundária a doenças sistêmicas e cirurgias prévias da coluna. ${ }^{5}$

É importante ressaltar seu efeito debilitante na saúde geral dos pacientes. Bess et $\mathrm{al}^{6}$ observaram que pacientes com deformidade vertebral têm maior prevalência de distúrbios psiquiátricos e pior qualidade de vida em relação àqueles com idade semelhante ou com doenças crônicas gerais, como diabetes e hipertensão arterial. Em situações de desequilíbrio coronal e sagital graves, a limitação é similar a pacientes com câncer, déficit visual ou uso restrito dos membros superiores e inferiores.

O número de pacientes operados com EA aumenta a cada ano, sendo atualmente a condição em cirurgia de coluna com o maior crescimento proporcional de procedimentos realizados. ${ }^{7}$ Alta complexidade cirúrgica, pacientes com condições clínicas frequentemente comprometidas e os altos custos em saúde são desafios presentes no seu tratamento.

\section{Fisiopatologia}

Acredita-se que a EA se inicie com a degeneração discal. Durante o envelhecimento, o disco intervertebral perde proteoglicanos pelo aumento da atividade das proteases com consequente diminuição da pressão osmótica e da hidratação discal. ${ }^{8}$ Foi demonstrado que a partir dos 15 anos de idade as lesões anulares são comuns e também comprometem a biomecânica discal. Este processo resulta na perda da altura discal e na incapacidade do disco de desempenhar seu papel estabilizador, acarretando sobrecarga da coluna posterior. ${ }^{9}$

A sobrecarga das articulações facetárias, em geral, ocorre de maneira assimétrica, o que contribui para a deformidade progressiva e aumenta a probabilidade de estenose central e foraminal associadas. Especialmente a rotação axial pode comprometer o complexo ligamentar da coluna levando a laterolistese. $^{9,10}$

Fatores como lipossubstituição da musculatura paravertebral, comprometimento discal pelo tabagismo, obesidade, herança genética, desenvolvimento de doenças neurodegenerativas e alterações do equilíbrio e mobilidade na senilidade estão relacionados com o desenvolvimento da EA. 3,10,11

Pensava-se que a osteoporose estaria implicada no desenvolvimento da EA, o que foi refutado pela maioria dos estudos atuais que sugerem uma prevalência de osteoporose similar entre os pacientes com EA e a população geral. Não foi observada correlação entre o grau de osteopenia e a magnitude da curva. Constatou-se que a densidade mineral óssea é maior no lado côncavo da curva e no fêmur ipsilateral quando comparados com o outro lado. ${ }^{12}$

\section{Apresentação Clínica}

A apresentação clínica é variável em razão da heterogeneidade dos pacientes. Sintomas de estenose central com claudicação neurogênica são relatados em até 90\% dos pacientes.
Os pacientes com escoliose degenerativa do adulto também apresentam alívio dos sintomas ao se sentarem sustentando o tronco com os membros superiores. ${ }^{9,13}$

A dor lombar está presente em entre 60 e $80 \%$ dos pacientes, principalmente no lado convexo da curva devido às alterações degenerativas e à fadiga muscular acarretada pelo desequilíbrio sagital/coronal. A radiculopatia em um ou múltiplos níveis acomete entre 47 e $78 \%$ dos pacientes em razão da hipertrofia facetária do lado côncavo da curva e da laterolistese. ${ }^{14}$

A alterações posturais em razão de uma deformidade fixa ou por mecanismos compensatórios são frequentes e devem ser avaliadas de rotina. Inclinação do tronco, assimetrias pélvicas e escapulares, hipo/hipercifose, grau de flexão e extensão dos quadris, joelhos e tornozelos durante o ortostatismo e deambulação e a avaliação da linha do horizonte são os principais pontos de análise. ${ }^{15}$

Nos pacientes com EA, as queixas álgicas, neurológicas e as limitações em atividades de vida diária são mais frequentes que nos pacientes adolescentes, grupo no qual a deformidade estética e a progressão da curva são os principais motivos de insatisfação. $^{16}$

A EA que ocorre em razão do processo degenerativo, a escoliose de novo, acomete similarmente ambos os gêneros, se inicia em geral aos 50 anos de idade e tem repercussões clínicas relevantes por volta de 70,5 anos de idade, afetando entre 32 e $68 \%$ dos indivíduos $>65$ anos de idade. ${ }^{10,11}$ Tipicamente, acomete a coluna lombar com ângulo de Cobb $<40^{\circ}$. A associação com laterolistese é frequente, sendo ocasional a presença de curvas torácicas compensatórias. ${ }^{10}$ Quando comparada com a EIA, a escoliose de novo apresenta curvas com menor angulação, entretanto com maior progressão $\left(1,64^{\circ} /\right.$ ano versus $0,82^{\circ} /$ ano $){ }^{17}$

A EIA ocorre em indivíduos com escoliose preexistente, principalmente do sexo feminino, em dois padrões principais. Um grupo apresenta progressão constante após a maturidade esquelética e o outro somente apresenta evolução da curva por volta da $4^{\underline{a}}$ e $5^{\mathrm{a}}$ décadas de vida, após a menopausa. ${ }^{17}$ Quando comparada com a escoliose de novo, a EIA apresenta menos estenose central, ângulos de Cobb maiores (média $>50^{\circ}$ ), as curvas torácicas compensatórias são mais frequentes, as taxas de laterolistese/espondilolistese e o desequilíbrio coronal/ sagital são menos comuns. ${ }^{14}$

Em relação à deformidade rotatória, ela é observada em toda a coluna lombar nos casos de EIA, enquanto na escoliose de novo, ela é limitada ao ápice da escoliose e acompanhada de laterolistese. ${ }^{14}$

\section{Avaliação radiográfica}

A radiografia panorâmica da coluna em ortostase é o exame primordial para o diagnóstico e classificação da EA. Apesar de historicamente a avaliação destes casos ser feita essencialmente no plano coronal, a importância do equilíbrio sagital foi constatada nas últimas décadas, sendo fundamental seu estudo de rotina. $^{18}$

A obtenção adequada das imagens radiográficas é crucial e deve evidenciar da base do crânio proximalmente às cabeças femorais distalmente. Sempre que possível, o paciente deve 
estar em ortostase sem a utilização de suportes para avaliação dos mecanismos compensatórios presentes. Para não deambuladores, recomenda-se a radiografia sentado.

A flexibilidade da deformidade ou a presença de curvas estruturadas podem ser avaliadas através de radiografias dinâmicas com inclinações ou tração. Esta informação pode ser útil no planejamento pré-operatório, prevendo quais técnicas serão requeridas para a correção adequada da deformidade e dificuldades intraoperatórias.

No plano coronal, recomenda-se mensurar o ângulo de Cobb de todas as curvas presentes, identificação das vértebras terminais, estável e neutra; avaliação do equilíbrio coronal pela distância entre uma linha de prumo em C7 e a linha vertical sacral central, além da obliquidade pélvica (caso presente, descartar uma possível discrepância de membros inferiores) ${ }^{10}$

No plano sagital ( - Figura 1), deve-se avaliar o equilíbrio sagital global pela distância entre uma linha de prumo em C7 e outra linha vertical passando pela borda posterosuperior de S1. Os parâmetros espinopélvicos, a lordose lombar e cifose torácica também devem ser avaliados. ${ }^{10}$

À análise radiográfica, o aumento da rotação da vértebra apical ( $\geq 3$ de Nash-Moe), ângulos de Cobb $>30^{\circ}$, assimetria dos discos intervertebrais acima e abaixo da vértebra apical, laterolistese $>6$ milímetros, linha interilíaca (linha entre os aspectos superiores de ambas as cristas ilíacas) seccionando L5 ao invés de L4 são preditivos de progressão da curva. Já a presença de osteófitos anteriores confere maior estabilidade à coluna vertebral. ${ }^{19}$
Tabela 1 Valores radiográficos preditivos de incapacidade pelo Índice de Oswestry - 40 pontos ${ }^{20}$

\begin{tabular}{|l|l|l|}
\hline $\begin{array}{l}\text { Parâmetro } \\
\text { radiográfico }\end{array}$ & $\begin{array}{l}\text { Ponto de corte } \\
\text { radiográfico }\end{array}$ & $\boldsymbol{r}$ \\
\hline IP-LL & $11^{\circ}$ & 0.45 \\
\hline VP & $22^{\circ}$ & 0.38 \\
\hline SVA & $46 \mathrm{~mm}$ & 0.47 \\
\hline
\end{tabular}

Abreviações: IP, incidência pélvica; LL, lordose lombar; VP, versão pélvica; SVA, eixo sagital vertical.

A avaliação dos parâmetros espinopélvicos é fundamental para a classificação do paciente e para o estabelecimento da melhor estratégia cirúrgica ( - Tabela 1 ). Valores de versão pélvica (VP) de $22^{\circ}$, eixo vertical sagital (SVA) de $46 \mathrm{~mm}$ e IPLL de $11^{\circ}$ são preditivos de incapacidade pelo Oswestry Disability Index (ODI) > 40 e são contemplados pelos modificadores sagitais da classificação de SRS-Schwab. ${ }^{20}$

$O$ estudo complementar por meio da tomografia computadorizada (TC) e da ressonância magnética (RM), apesar de não identificar fatores dinâmicos da ortostase, é benéfico por possibilitar a reprodução de imagens em três dimensões (3D) e detalhamento dos componentes ósseos e de partes moles intra e extravertebrais. A TC detalha melhor as características ósseas, incluindo artrose facetária, rotação vertebral e diâmetro do pedículo. Por outro lado, a RM fornece informações sobre o comprometimento discal, estenose central e foraminal além da lipossubstituição da musculatura paravertebral. ${ }^{15}$
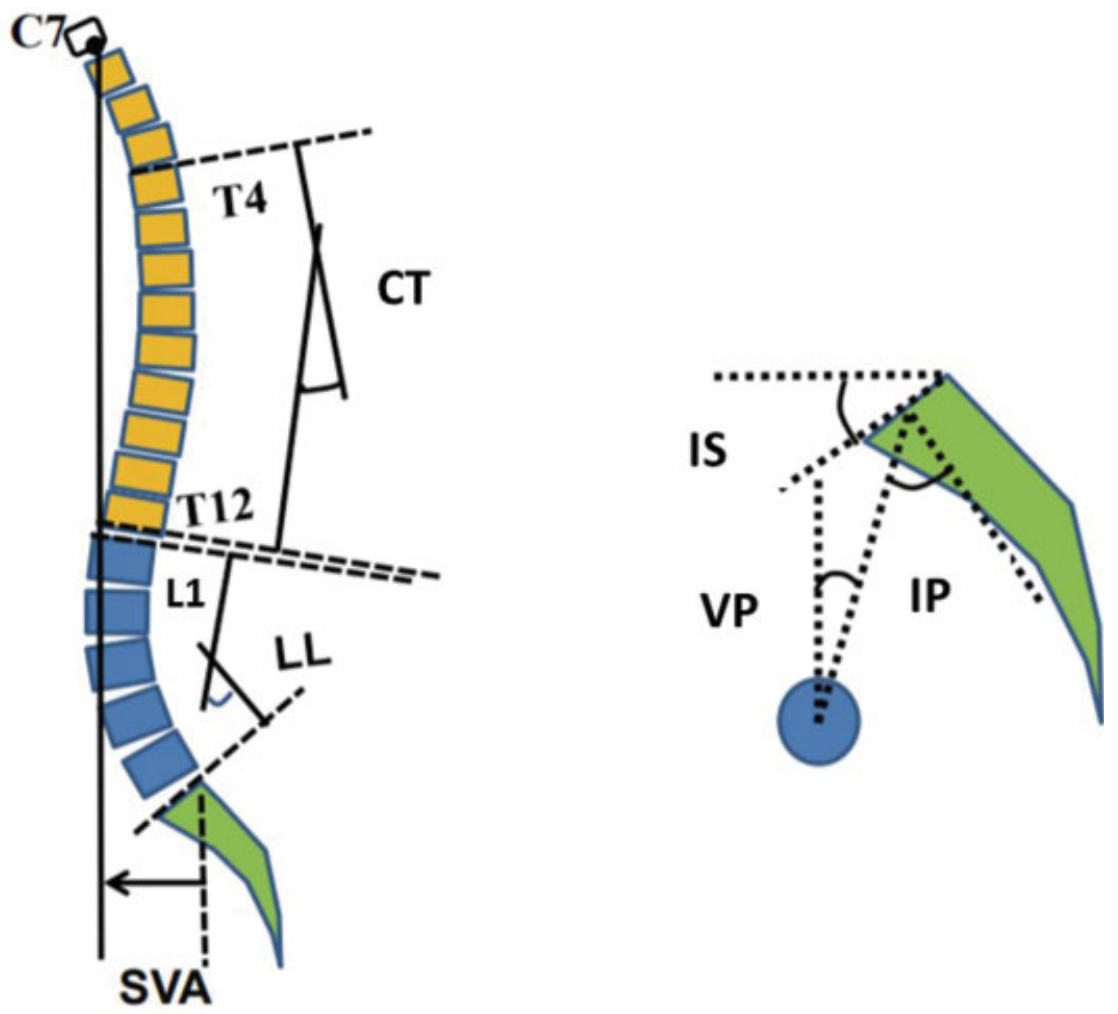

Fig. 1 SVA: Eixo Vertical Sagital; LL: lordose lombar; CT: cifose torácica. Parâmetros espinopélvicos - IS: Inclinação Sacral; VP: versão pélvica. IP: incidência pélvica. 
Os aparelhos de imagem biplanar com capacidade tridimensional configuram um método diagnóstico relativamente novo e promissor com menores taxas de radiação. Fornecem imagens de todo o corpo, incluindo cabeça, coluna, pelve e membros inferiores, otimizando a análise do equilíbrio global do paciente. ${ }^{21}$

\section{Classificações}

A classificação atualmente utilizada, em razão de seu poder descritivo, reprodutibilidade e correlação com qualidade de vida, é a de SRS-Schwab ( - Figura 2).$^{20}$ Inicialmente, deve-se mensurar o ângulo de Cobb no plano coronal com caracterização da deformidade. Posteriormente, a avaliação do plano sagital é realizada através da análise dos três modificadores sagitais.

A presença de escore "+ " ou "++" em qualquer um dos modificadores sagitais é preditiva de pior performance clínica, conforme referido previamente.

Em razão da grande variedade de osteotomias e terminologias empregadas no tratamento da EA, existe uma importante limitação na comparação de resultados entre as diferentes técnicas. Com o propósito de superar este problema, Schwab et al propuseram uma classificação anatômica de osteotomias baseada no local da ressecção (- Figura 3). ${ }^{22}$

As artrodeses intersomáticas por vias anterolaterais ganharam popularidade na última década. Apesar de iniciaImente terem sido empregadas para descompressão indireta, passaram a fazer parte do arsenal terapêutico das deformi- dades do adulto. O procedimento de realinhamento da coluna anterior (RCA) consiste no acréscimo de secção do ligamento longitudinal anterior (LLA) a tais técnicas, aumentando seu poder de correção. ${ }^{23}$

Os procedimentos de RCA, por proporcionarem a mobilidade das três colunas de Dennis, possibilitaram tratamentos minimamente invasivos para a correção de deformidades do adulto em casos historicamente tratados apenas através de osteotomias. $^{23}$

Uribe et al, com o intuito de facilitar a comunicação entre os cirurgiões e padronizar as intervenções de RCA em pesquisas clínicas, utilizaram como base a classificação anatômica de osteotomias, e propuseram uma nova classificação (-Figura 4). ${ }^{23}$

O grau A é único no qual a correção se dá por abordagem anterior ou lateral, sem osteotomias posteriores. Nestes casos, a correção angular é alcançada através da secção do LLA e da lordose do espaçador discal. Aproximadamente, se atinge $7,8^{\circ}$ de lordose por segmento com cages de $30^{\circ} .^{23}$ Os demais graus se baseiam nas osteotomias posteriores realizadas (modificador de Schwab) e por qual via foi realizado o procedimento de RCA (modificador de abordagem).

\section{Tratamento}

\section{Tratamento Clínico}

Apesar da ausência de evidências científicas consistentes que justifiquem sua indicação, os métodos não cirúrgicos são geralmente a primeira linha de tratamento. São raros os

\section{Tipos de curvas no plano coronal}

\section{Modificadores sagitais}
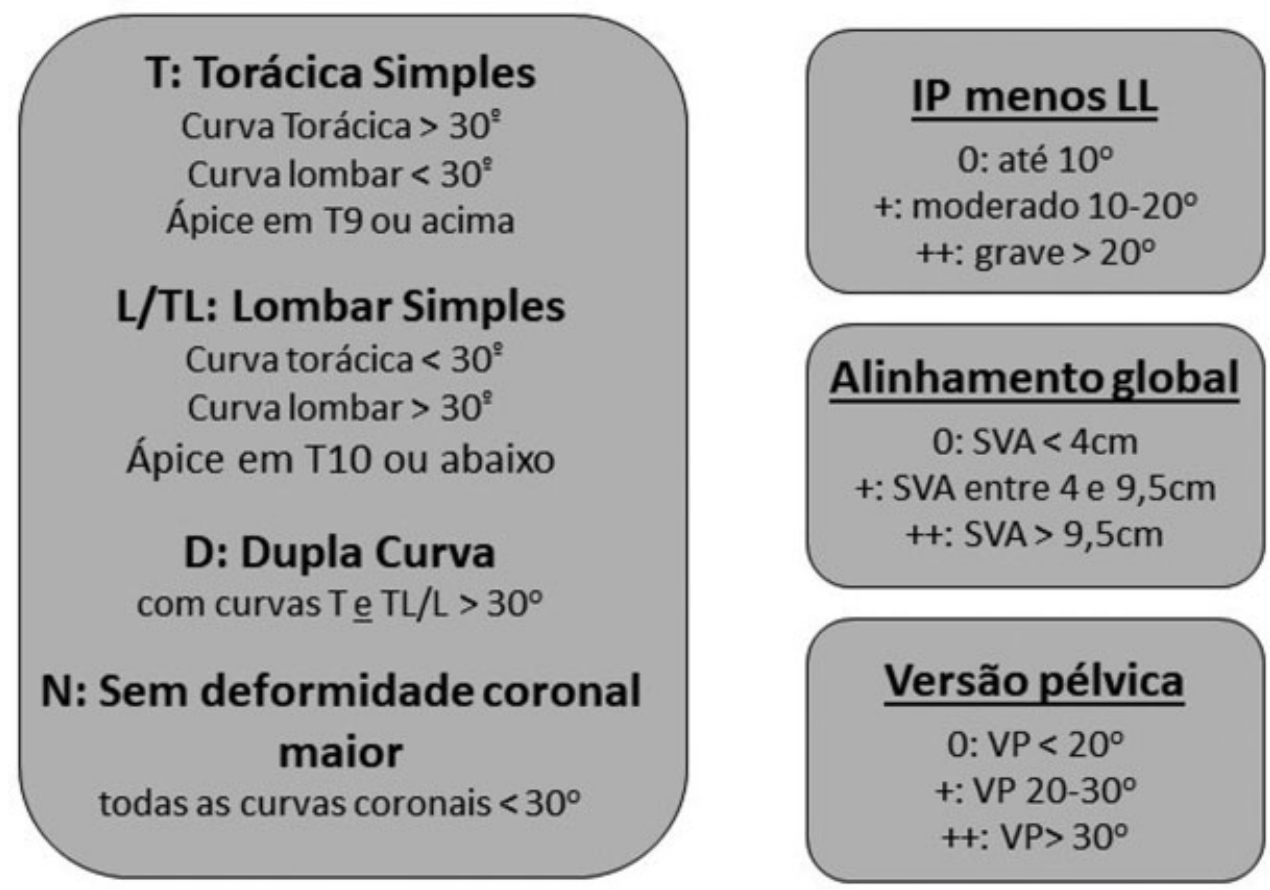

Fig. 2 Classificação de SRS-Schwab. ${ }^{20}$ IP: incidência pélvica; LL: lordose lombar; SVA: eixo vertical sagital; VP: versão pélvica. 


\section{1}

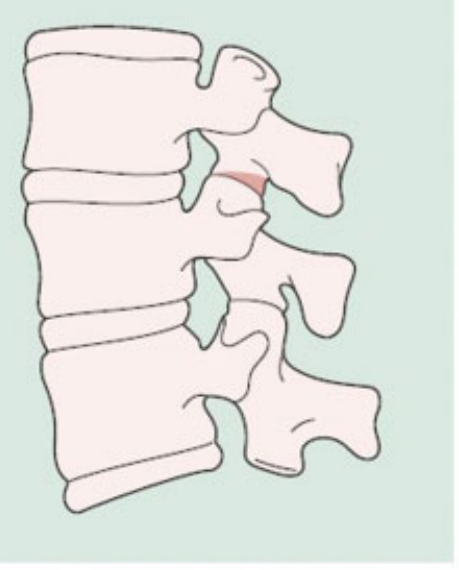

4

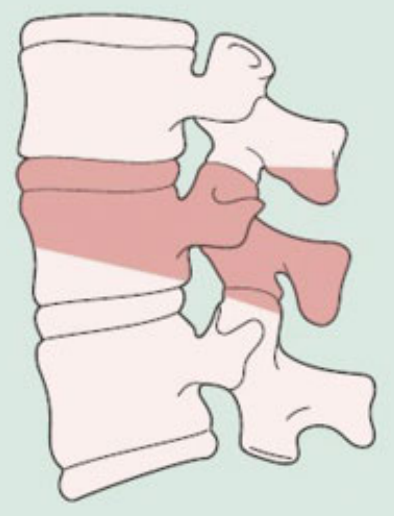

2

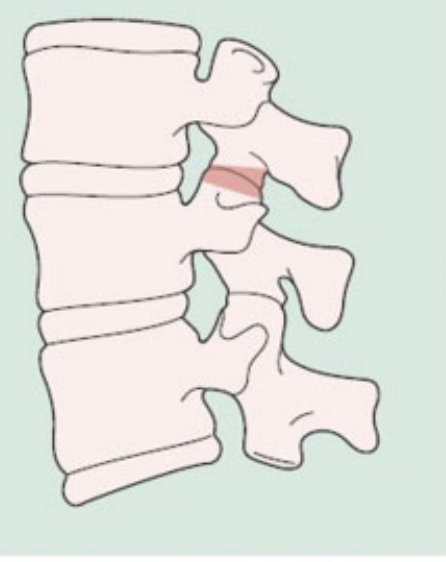

5

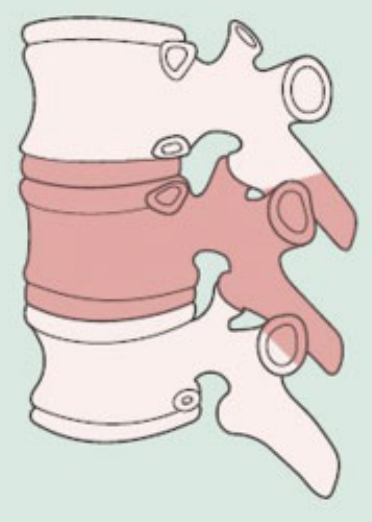

3

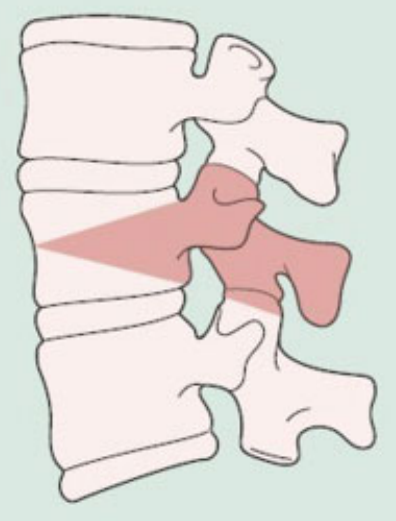

6

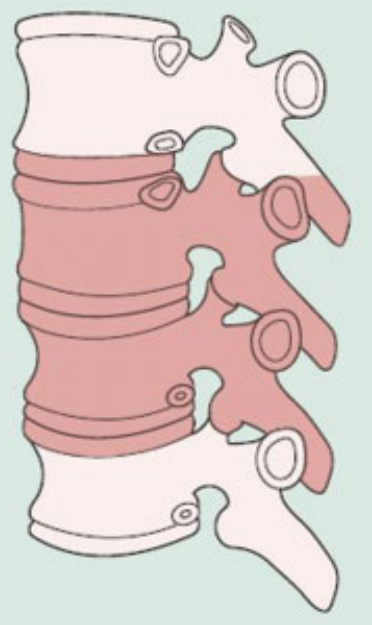

Fig. 3 Classificação anatômica de Osteotomias por Schwab et al. ${ }^{22}$ Grau 1: Parcial das facetas. Grau 2: Total das facetas. Grau 3: Pedículo e parcial do corpo. Grau 4: Pedículo, parcial do corpo e disco. Grau 5: Completa de disco e corpo. Grau 6: Múltiplas vértebras e discos.

estudos que recomendam fisioterapia, alongamentos, terapia manual e calor local para pacientes com EA, nível de evidência IV. ${ }^{24}$ Em casos de dor crônica, acupuntura e terapia cognitivo-comportamental podem ser consideradas. $\mathrm{O}$ uso de coletes nestes pacientes não se mostrou efetivo. ${ }^{25}$

O tratamento medicamentoso é baseado em analgésicos, anti-inflamatórios não esteroidais, anticonvulsivantes e antidepressivos. A avaliação da densidade mineral óssea deve ser realizada de rotina e deve ser iniciado tratamento específico, caso diagnosticada osteoporose. As infiltrações epidurais, de ponto gatilho ou bloqueios de nervos periféricos, são benéficas como provas terapêuticas e proporcionam alívio da dor em curto e médio prazo, embora ainda sejam necessários estudos sobre seus efeitos a longo prazo. ${ }^{26}$

Os pacientes que apresentam os melhores resultados com tratamento clínico são aqueles com deformidades leves, não progressivas e pouco sintomáticos. Em um estudo multicêntrico, Passias et al. ${ }^{27}$ comparando os tratamentos clínico, cirúrgico e o clínico-para-cirúrgico (crossover) observaram que os participantes que optaram por cirurgia apresentavam mais limitação e piores desempenhos em escores de dor/qualidade de vida.

\section{Tratamento cirúrgico}

As principais indicações cirúrgicas são limitação em atividades de vida diária, dor, sintomas neurológicos, confirmação da progressão da curva e falha do tratamento conservador. ${ }^{25}$ Em termos radiográficos, preconiza-se a intervenção cirúrgica em curvas lombares $>$ de $30^{\circ}$ a $40^{\circ}, 6 \mathrm{~mm}$ de laterolistese, progressão da curva em $>10^{\circ}$ ou da subluxação em $>3 \mathrm{~mm} .{ }^{9}$

Os principais pontos a serem considerados na indicação cirúrgica são o reestabelecimento dos parâmetros de equilíbrio global (principalmente o sagital) e a descompressão neurológica. A elevada prevalência de comorbidades nesta população, a complexidade cirúrgica e a expectativa do paciente sobre seu tratamento também devem ser ponderados.

A maioria dos estudos em deformidade do adulto comparando o tratamento clínico e cirúrgico favorecem a intervenção cirúrgica. Os pacientes operados apresentam melhora estatisticamente significativa em qualidade de vida, dor, performance e menor possibilidade de deterioração clínica em relação aos que receberam tratamento conservador. ${ }^{28}$ 

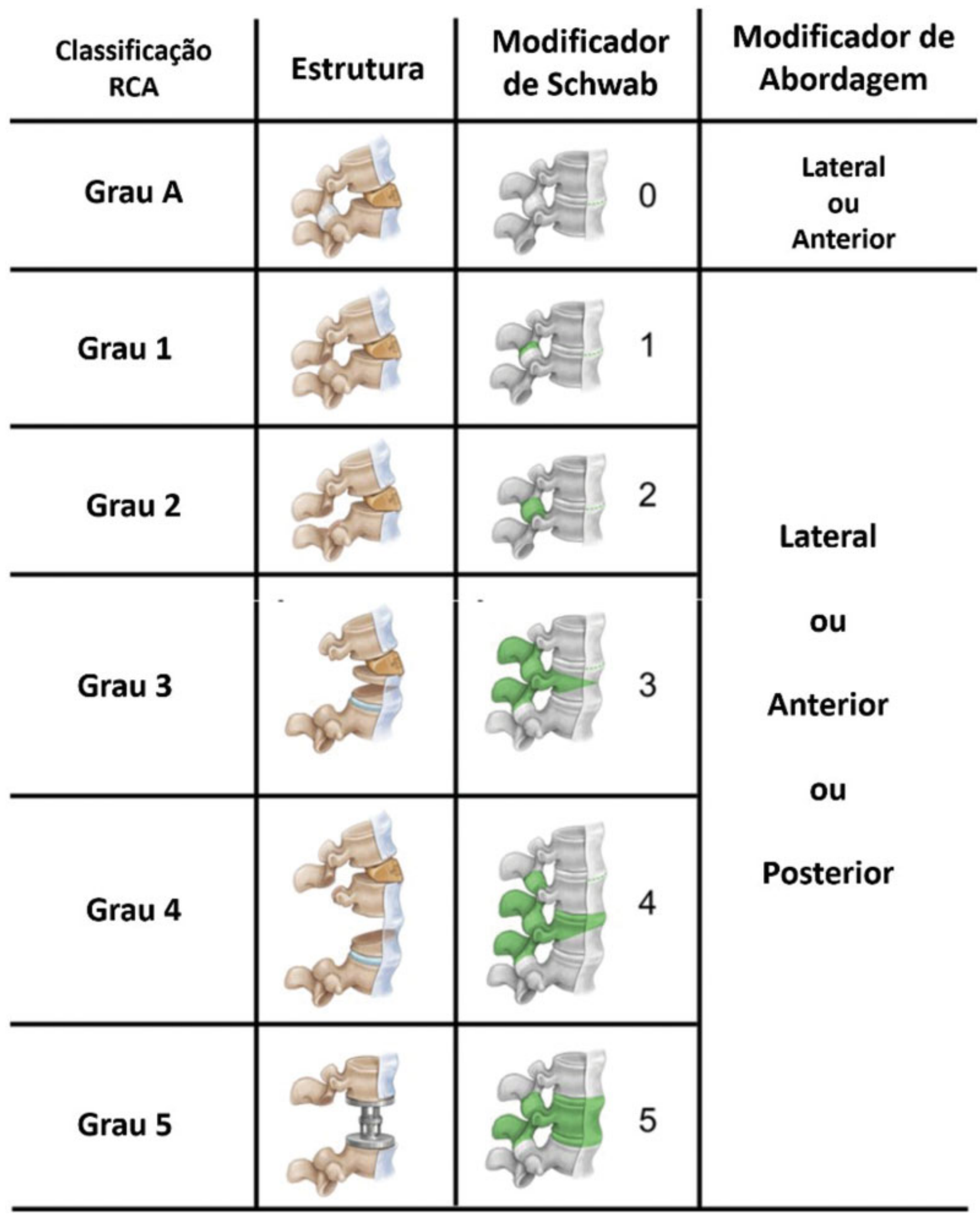

Fig. 4 Classificação dos procedimentos de realinhamento da coluna anterior. ${ }^{23}$

Quando o tratamento cirúrgico é escolhido, existem diversas técnicas que foram subdivididas em seis grupos de forma hierarquizada por Silva et $\mathrm{al}^{9}$ : descompressão isolada; descompressão com artrodese posterolateral curta; descompressão com artrodese posterolateral da coluna lombar; descompressão com artrodese anterior e posterolateral; extensão da artrodese e instrumentação à coluna torácica; inclusão de osteotomias para correção de deformidades.

A descompressão isolada é melhor indicada em pacientes com sintomas neurológicos, sem dor axial e com curvas lombares estáveis ( $\mathrm{Cobb}<30^{\circ},<2 \mathrm{~mm}$ de laterolistese e presença de osteófitos anteriores). ${ }^{14}$ Em casos similares 
com necessidade de descompressão ampla e possibilidade de instabilidade, se o equilíbrio sagital e coronal estiverem preservados, a artrodese apenas do segmento abordado deve ser considerada. ${ }^{29}$

Em pacientes com risco de pseudoartrose e programação de artrodese longa, a associação de artrodese intersomática é benéfica, sobretudo em níveis lombares baixos, caso seja realizada fusão lombossacra. ${ }^{9} \mathrm{O}$ emprego de técnicas de acesso anterior e lateral, além de proporcionarem descompressão direta e indireta pela distração foraminal, permitem o uso de espaçadores discais com maior área, menor risco de fratura dos platôs (subsidência) e com potencial de correção angular nos planos sagital e coronal. ${ }^{10,29}$

Na presença de desequilíbrio global importante associado a aumento da cifose torácica, a extensão da artrodese à coluna torácica pode ser necessária e reduz a chance de cifose juncional proximal. Prefere-se a fusão ao nível de T10 ou cefálicos em razão da estabilidade conferida pela articulação das respectivas costelas ao esterno nestes níveis (costelas verdadeiras), o que não ocorre com as costelas flutuantes (T11 e T12). ${ }^{9,30}$

Os casos que apresentam deformidades rígidas $(<30 \%$ de correção em radiografias dinâmicas) ou artrodese prévia com desequilíbrio sagital importante são elegíveis para osteotomias. As osteotomias, além de restaurarem o equilíbrio vertebral, diminuem a sobrecarga sobre a interface osso/ material de síntese e a chance de falha mecânica. ${ }^{9}$ Embora estes procedimentos aumentem o tempo cirúrgico, o sangramento e a morbidade perioperatória, a correção do equilíbrio sagital se mostrou como o fator isolado com melhor impacto nos resultados pós-operatórios. ${ }^{19}$

Como princípios de escolha dos níveis de artrodese, são recomentados ${ }^{19}$ :

$\S$ Não pare no ápice da curva

$\S$ Não pare em pontos de cifose

$\S$ Inclua níveis com subluxação lateral importante

$\S$ Inclua espondilolistese ou retrolistese

$\S$ A vértebra superior instrumentada deve, idealmente, ser horizontal

§ A fixação do ilíaco deve ser fortemente considerada em artrodeses longas

\section{Técnicas Cirúrgicas}

Comparando-se as técnicas de artrodese posterolateral (APL) e a artrodese lombar intersomática transforaminal (TLIF), constatou-se que a APL apresenta menor tempo cirúrgico, sangramento e complicações pós-operatórias. No entanto, a TLIF apresentou melhor correção sagital e melhores resultados clínicos em relação a dor e satisfação. ${ }^{31}$ Em termos gerais, cirurgias com artrodese intersomática para EA apresentam melhores resultados clínicos que a APL estrita. ${ }^{32}$

A artrodese intersomática por via anterior (ALIF) apresenta a vantagem de liberação anterior, otimização da descompressão com colocação de maiores implantes intervertebrais, possibilitando maiores correções sagitais e menores taxas de subsidência quando comparada às técnicas por via posterior. ${ }^{33}$ A técnica é adequada paras os níveis L4-L5 e sobretudo L5-S1 em razão da anatomia vascular local. A lesão vascular e visceral além de ejaculação retrógrada são complicações possíveis. ${ }^{32}$
A artrodese intersomática por via lateral (LLIF) ganhou popularidade nos últimos anos e permite correção de T12L1 a L4-L5 nos planos sagital e coronal com implantes robustos como na ALIF e maior facilidade cirúrgica em pacientes com cirurgias abdominais prévias ou obesos. Como desvantagens, há a dificuldade em se acessar o disco L5-S1 devido à presença da crista ilíaca, a longa curva de aprendizado desta técnica, o risco de fraqueza do psoas e da lesão do plexo lombar. ${ }^{19}$

Em um estudo multicêntrico prospectivo com 107 pacientes submetidos a LLIF por escoliose degenerativa do adulto, Philips et $\mathrm{al}^{34}$ relataram melhora nos escores de ODI, escala visual analógica (EVA) e no questionário de qualidade de vida SF-36. A média do ângulo de Cobb passou de $20.9^{\circ}$ para $13,5^{\circ}$ no pós-operatório, e se manteve em $15,2^{\circ}$ após 2 anos de seguimento. Nos pacientes com redução da lordose lombar, esse parâmetro passou de $27,7^{\circ}$ para $33,6^{\circ}$ após dois anos.

Em razão do tempo cirúrgico prolongado e altas taxas de morbidade perioperatória pelos procedimentos clássicos, há um esforço atual no desenvolvimento de técnicas minimamente invasivas (MIS), com resultados preliminares encorajadores.

\section{Complicações}

Em uma revisão de casos realizada pela Sociedade de Pesquisa em Escoliose (SRS, na sigla em inglês) a taxa de complicações cirúrgicas na EA é de 13.4\%, embora outros estudos relatem até $40 \%$. Lesão de dura-máter, falha do implante, infecção superficial e profunda da ferida operatória e déficits neurológicos são as lesões mais frequentes. ${ }^{35}$ Pacientes obesos, tabagistas, osteoporóticos e com idade avançada (> 65 anos) estão sob risco adicional. $^{11}$

A cifose juncional proximal ocorre em entre 20 e $40 \%$ dos pacientes e pode se apresentar de forma precoce ou tardia. Os índices de pseudoartrose com as técnicas atuais são menores, estudos recentes apontam sua ocorrência em entre 4 e $24 \%$ dos casos. As taxas de reoperação vão de $16.7 \%$ com 90 dias a $40 \%$ em 11 anos. $^{11}$

Dadas as características clínicas deste grupo, as complicações sistêmicas como infarto agudo do miocárdio, pneumonia, íleo adinâmico, trombose venosa profunda e infecção do trato urinário não são incomuns.

\section{Considerações Finais}

A escoliose degenerativa do adulto é uma doença potencialmente limitante que acomete um grupo heterogêneo de pacientes com importantes limitações clínicas. 0 tratamento clínico se mostrou pouco efetivo e a indicação cirúrgica é frequente. Representa um desafio aos cirurgiões de coluna por sua complexidade e aumento de prevalência.

A avaliação clínica e radiológica deve ser criteriosa para se determinar a real origem dos sintomas. A descompressão das estruturas neurológicas e a manutenção do equilíbrio sagital e coronal, sempre que possível, devem ser os principais objetivos do tratamento.

O desenvolvimento de técnicas minimamente invasivas já é realidade, e seu aperfeiçoamento poderá reduzir a 
incidência de complicações pós-operatórias e também fornecer melhores resultados. Neste sentido, novos estudos são necessários para se atestar o real benefício destes procedimentos no tratamento da EA.

\section{Nota}

O artigo submetido é "Artigo de Atualização" não sendo necessária aprovação do Comitê de Ética em pesquisa.

Os autores atestam que o estudo foi realizado sem a participação direta de humanos.

Trabalho desenvolvido na Divisão de Cirurgia de Coluna Vertebral, Departamento de Ortopedia e Traumatologia, Hospital das Clinicas HCFMUSP, Faculdade de Medicina, Universidade de São Paulo, São Paulo, SP, Brasil.

\section{Conflito De Interesses}

Os autores declaram não haver conflito de interesses.

\section{Referências}

1 Wong LL, Carvalho JA. O rápido processo de envelhecimento populacional do Brasil: sérios desafios para as políticas públicas. Rev Bras Estud Popul 2006;23(01):5-26

2 Nasri F. O envelhecimento populacional no Brasil. Einstein (Sao Paulo) 2008;6(Suppl 1):S4-S6

3 Schwab F, Dubey A, Gamez L, et al. Adult scoliosis: prevalence, SF-36, and nutritional parameters in an elderly volunteer population. Spine (Phila Pa 1976) 2005;30(09):1082-1085

4 Aebi M. The adult scoliosis. Eur Spine J 2005;14(10):925-948

5 Youssef JA, Orndorff DO, Patty CA, et al. Current status of adult spinal deformity. Global Spine J 2013;3(01):51-62

6 Bess S, Line B, Fu KM, et al; International Spine Study Group. The Health Impact of Symptomatic Adult Spinal Deformity: Comparison of Deformity Types to United States Population Norms and Chronic Diseases. Spine (Phila Pa 1976) 2016;41(03):224-233

7 McCarthy I, Hostin R, O'Brien M, Saigal R, Ames CP. Health economic analysis of adult deformity surgery. Neurosurg Clin $\mathrm{N}$ Am 2013;24(02):293-304

8 Vernon-Roberts B, Moore RJ, Fraser RD. The natural history of agerelated disc degeneration: the influence of age and pathology on cell populations in the L4-L5 disc. Spine (Phila Pa 1976) 2008;33 (25):2767-2773

9 Silva FE, Lenke LG. Adult degenerative scoliosis: evaluation and management. Neurosurg Focus 2010;28(03):E1

10 York PJ, Kim HJ. Degenerative Scoliosis. Curr Rev Musculoskelet Med 2017;10(04):547-558

11 Diebo BG, Shah NV, Boachie-Adjei O, et al. Adult spinal deformity. Lancet 2019;394(10193):160-172

12 Routh RH, Rumancik S, Pathak RD, Burshell AL, Nauman EA. The relationship between bone mineral density and biomechanics in patients with osteoporosis and scoliosis. Osteoporos Int 2005;16 (12):1857-1863

13 Grubb SA, Lipscomb HJ, Suh PB. Results of surgical treatment of painful adult scoliosis. Spine (Phila Pa 1976) 1994;19(14):16191627

14 Cho KJ, Kim YT, Shin SH, Suk SI. Surgical treatment of adult degenerative scoliosis. Asian Spine J 2014;8(03):371-381

15 Smith JS, Shaffrey CI, Fu KM, et al. Clinical and radiographic evaluation of the adult spinal deformity patient. Neurosurg Clin N Am 2013;24(02):143-156

16 Bess S, Boachie-Adjei O, Burton D, et al. International Spine Study Group. Pain and disability determine treatment modality for older patients with adult scoliosis, while deformity guides treatment for younger patients. Spine (Phila Pa 1976) 2009;34(20):2186-2190
17 Marty-Poumarat C, Scattin L, Marpeau M, Garreau de Loubresse C, Aegerter P. Natural history of progressive adult scoliosis. Spine (Phila Pa 1976) 2007;32(11):1227-1234

18 Schwab FJ, Blondel B, Bess S, et al. International Spine Study Group (ISSG). Radiographical spinopelvic parameters and disability in the setting of adult spinal deformity: a prospective multicenter analysis. Spine (Phila Pa 1976) 2013;38(13):E803-E812

19 Kim W, Porrino JA, Hood KA, Chadaz TS, Klauser AS, Taljanovic MS. Clinical Evaluation, Imaging, and Management of Adolescent Idiopathic and Adult Degenerative Scoliosis. Curr Probl Diagn Radiol 2019;48(04):402-414

20 Schwab F, Ungar B, Blondel B, et al. Scoliosis Research SocietySchwab adult spinal deformity classification: a validation study. Spine (Phila Pa 1976) 2012;37(12):1077-1082

21 Somoskeöy S, Tunyogi-Csapó M, Bogyó C, Illés T. Accuracy and reliability of coronal and sagittal spinal curvature data based on patient-specific three-dimensional models created by the EOS 2D/3D imaging system. Spine J 2012;12(11):1052-1059

22 Schwab F, Blondel B, Chay E, et al. The comprehensive anatomical spinal osteotomy classification. Neurosurgery 2014;74(01): $112-120$

23 Uribe JS, Schwab F, Mundis GM, et al. The comprehensive anatomical spinal osteotomy and anterior column realignment classification. J Neurosurg Spine 2018;29(05):565-575

24 Teles AR, Mattei TA, Righesso O, Falavigna A. Effectiveness of Operative and Nonoperative Care for Adult Spinal Deformity: Systematic Review of the Literature. Global Spine J 2017;7(02): 170-178

25 Haldeman S, Nordin M, Chou R, et al. The Global Spine Care Initiative: World Spine Care executive summary on reducing spine-related disability in low- and middle-income communities. Eur Spine J 2018;27(Suppl 6):776-785

26 DePalma MJ, Slipman CW. Evidence-informed management of chronic low back pain with epidural steroid injections. Spine J 2008;8(01):45-55

27 Passias PG, Jalai CM, Line BG, et al. International Spine Study Group. Patient profiling can identify patients with adult spinal deformity (ASD) at risk for conversion from nonoperative to surgical treatment: initial steps to reduce ineffective ASD management. Spine J 2018;18(02):234-244

28 Sengupta D. Adult Spinal Deformity. In: Rao R, Smuck M, editors. Orthopaedic knowledge update: spine. 4th ed. Rosemont, IL: American Academy of Orthopaedic Surgeons; 2012:349-367

29 Cho KJ, Suk SI, Park SR, et al. Short fusion versus long fusion for degenerative lumbar scoliosis. Eur Spine J 2008;17(05):650-656

$30 \mathrm{Fu}$ X, Sun XL, Harris JA, et al. Long fusion correction of degenerative adult spinal deformity and the selection of the upper or lower thoracic region as the site of proximal instrumentation: a systematic review and meta-analysis. BMJ Open 2016;6(11):e012103

31 Li FC, Chen QX, Chen WS, Xu K, Wu QH, Chen G. Posterolateral lumbar fusion versus transforaminal lumbar interbody fusion for the treatment of degenerative lumbar scoliosis. J Clin Neurosci 2013;20(09):1241-1245

32 Mobbs RJ, Phan K, Malham G, Seex K, Rao PJ. Lumbar interbody fusion: techniques, indications and comparison of interbody fusion options including PLIF, TLIF, MI-TLIF, OLIF/ATP, LLIF and ALIF. J Spine Surg 2015;1(01):2-18

33 Rao PJ, Loganathan A, Yeung V, Mobbs RJ. Outcomes of anterior lumbar interbody fusion surgery based on indication: a prospective study. Neurosurgery 2015;76(01):7-23

34 Phillips FM, Isaacs RE, Rodgers WB, et al. Adult degenerative scoliosis treated with XLIF: clinical and radiographical results of a prospective multicenter study with 24-month follow-up. Spine (Phila Pa 1976) 2013;38(21):1853-1861

35 Sansur CA, Smith JS, Coe JD, et al. Scoliosis research society morbidity and mortality of adult scoliosis surgery. Spine (Phila Pa 1976) 2011;36(09):E593-E597 